Time spectrum of a coherent pulse propagating through a single resonant medium

This content has been downloaded from IOPscience. Please scroll down to see the full text. 2015 EPL 11134003

(http://iopscience.iop.org/0295-5075/111/3/34003)

View the table of contents for this issue, or go to the journal homepage for more

Download details:

IP Address: 131.169.38.71

This content was downloaded on 23/02/2016 at 08:19

Please note that terms and conditions apply. 


\title{
Time spectrum of a coherent pulse propagating through a single resonant medium
}

\author{
Y. Friedman, J. M. Steiner and E. Yudkin \\ Jerusalem College of Technology - Jerusalem, Israel
}

received 3 June 2015; accepted in final form 1 August 2015

published online 25 August 2015

PACS 42.25.Bs - Wave propagation, transmission and absorption

PACS $61.05 . c c-$ Theories of x-ray diffraction and scattering

PACS 33.45. $+\mathrm{x}-$ Mössbauer spectra

\begin{abstract}
A new formula for the delayed electromagnetic field and the time spectrum of a coherent synchrotron radiation pulse propagating through a single resonant medium is obtained. Unlike the commonly accepted one-term formula for such a time spectrum, our formula contains an additional term arising from the secondary radiation. This term helps to overcome some shortcomings of the accepted formula. Using the experimental data from the time spectrum of an enriched stainless-steel foil obtained recently at PETRA III (DESY, Hamburg) as a test case, our new formula provides a quality fit. Unlike the commonly available computer programs used by experimentalists, our fit does not require the averaging of the thickness parameter.
\end{abstract}

Copyright (C) EPLA, 2015

Introduction. - In order to properly describe the propagation of an electromagnetic wave through a resonant medium, one must first understand the interaction of the electromagnetic wave with the vibrating charges in the medium. This is best understood when there exists only a single resonant frequency of the vibrating charges in the range of the frequencies of the wave. A good example of this is the propagation of a synchrotron radiation (SR) x-ray pulse through a single resonant medium containing ${ }^{57} \mathrm{Fe}$.

After the discovery of the Mössbauer effect, several papers have described the time delay of a coherent pulse propagating through a ${ }^{57} \mathrm{Fe}$ target. The only indicator for the delay of a SR pulse by a target, is the experimentally measured intensity of the exiting delayed field emerging from the back of the target as a function of the delay time - the time spectrum.

By applying classical linear response theory, Lynch et al. [1] were the first to predict the time spectrum $I(\tau)$ of an external exponentially decaying exciting pulse of radiation which performs driven oscillations of the vibrating nuclei in such a target

$$
I(\tau) \propto e^{-\tau} J_{0}^{2}(\sqrt{T \tau}),
$$

where $\tau$ is the dimensionless time delay (the time delay divided by the lifetime of the excited level of the nuclei), $T$ is the effective thickness of the target and $J_{0}$ is the
Bessel function of the first kind of order zero. A similar formula appears also in the work by Smirnov [2].

Using the same classical methods, Kagan et al. [3] obtained

$$
I(\tau) \propto e^{-\tau} T^{2} \frac{J_{1}^{2}(\sqrt{T \tau})}{T \tau}
$$

for the delayed transmission of a SR pulse, where $J_{1}$ is the Bessel function of the first kind of order one.

By considering the vibrating nuclei in the medium (target) as harmonic oscillators with a single natural frequency and solving simultaneously the forced harmonic-oscillator equation together with Maxwell's equations, Burnham and Chiao [4], in the late sixties, described the propagation of a coherent exciting pulse through a single resonant medium assuming infinite lifetimes. Their solution for the exiting electromagnetic field also predicts the time spectrum (2).

Formulae for the time spectrum of SR through a single resonant medium similar to formula (2) were derived by several authors, see [5]. A comprehensive review of coherent pulse propagation through a single resonant medium may be found in [6].

In this paper we extend the analysis of [4] for a finite lifetime. Moreover, we release their assumption that the amplitude of the delayed field is real. Indeed, one may assume that the amplitude of the primary radiation field generated by the vibrations of the nuclei caused by the exciting 
pulse is real. The primary field is the main contribution to the delayed field. However, one must also consider a purely imaginary amplitude associated with the secondary radiation field generated by the vibrations of the resonant nuclei caused by this primary field. In order to describe this field we took into consideration the existence of coherent backward scattering, observed experimentally and justified theoretically (see, for example, [7] and [8]).

Our analysis predicts the time spectrum

$$
I(\tau) \propto e^{-\tau} T^{2}\left(\frac{J_{1}^{2}(\sqrt{T \tau})}{T \tau}+\alpha^{2} J_{0}^{2}(\sqrt{T \tau})\right)
$$

for some constant $\alpha$ depending on the medium. We further show that this prediction is supported by experimental data. Unlike the commonly available computer programs MOTIF [9] and CONUSS [10] currently used by experimentalists to fit the time spectrum, our formula avoids the need for averaging the thickness parameter.

The interaction of an electromagnetic field and oscillating currents in a single resonant medium. Our derivation of the equations for the interaction of an electromagnetic field and oscillating currents in a single resonant medium is based on [4]. Denote by $\omega_{0}$ the nuclear resonant frequency of the medium. Suppose that the electromagnetic field of a coherent, linearly polarized SR exciting pulse with frequency close to this resonant frequency propagating in the positive $z$-direction, enters the medium at the point $z=0$. Since the polarization is linear we may assume that the electromagnetic field $E(t, z)$ is a scalar valued function of time $t$ and position $z$. The time $t=0$ is the time when the end of this pulse enters the medium. Most of this pulse is non-scattered and is unaffected by the medium. We consider only the delayed (scattered) part of the pulse.

The motion of the vibrating nuclei is described as a harmonic oscillator with natural frequency $\omega_{0}$, damping rate $\gamma$ with an external force caused by the action of the electromagnetic field on the vibrating nuclei. The equation for the charge (nuclear) displacement $X(t, z)$ in the direction of the polarisation is

$$
\frac{\partial^{2} X}{\partial t^{2}}+\gamma \frac{\partial X}{\partial t}+\omega_{0}^{2} X=\frac{q}{m} E,
$$

where $q, m$ denote the charge and mass of the vibrating nuclei, respectively.

The vibrating nuclei generate in turn an electromagnetic current. Using Maxwell's equations in a medium with no free charges, the interaction of this current with the field $E(t, z)$ is described by

$$
\frac{\partial^{2} E}{\partial z^{2}}-\frac{1}{c^{2}} \frac{\partial^{2} E}{\partial t^{2}}=\mu_{0} \rho_{e} \frac{\partial^{2} X}{\partial t^{2}}
$$

where $\mu_{0}$ and $\rho_{e}$ denote the magnetic permeability and the charge density of the excited resonant nuclei, respectively.
The coupled equations (4) and (5) describe the interaction of an electromagnetic field and oscillating currents in a single resonant medium.

In nuclear resonant scattering, only frequencies very close to the natural frequency $\omega_{0}$ of the medium contribute to the scattering. Hence, using the well-known slow-varying amplitude approximation, we can represent the charge displacement $X(t, z)$ and the electromagnetic field $E(t, z)$ as

$$
X(t, z)=e^{i \omega_{0}\left(t-\frac{z}{c}\right)} \mathcal{X}(t, z), \quad E(t, z)=e^{i \omega_{0}\left(t-\frac{z}{c}\right)} \mathcal{E}(t, z),
$$

where their amplitudes $\mathcal{X}(t, z)$ and $\mathcal{E}(t, z)$ are varying slowly (in time and in space) during an optical period or over the distance of an optical wavelength. It should be emphasized that since the frequency and wavelength of the field inside the medium differ from $\omega_{0}$ and $c \omega_{0}$, respectively, we must treat the amplitudes $\mathcal{X}(t, z)$ and $\mathcal{E}(t, z)$ as complex-valued functions.

Following the well-known technique (see, for example, [11]) this approximation reduces the second-order coupled equations (4) and (5) to the first-order coupled equations

$$
\frac{\partial \mathcal{X}(t, z)}{\partial t}+\frac{\gamma}{2} \mathcal{X}(t, z)=\frac{-i q}{2 m \omega_{0}} \mathcal{E}(t, z)
$$

and

$$
\frac{\partial \mathcal{E}(t, z)}{\partial z}+\frac{1}{c} \frac{\partial \mathcal{E}(t, z)}{\partial t}=\frac{-i \omega_{0} c \mu_{0} \rho_{e}}{2} \mathcal{X}(t, z)
$$

for the complex amplitudes.

Time delay. - We introduce the time delay $t^{\prime}=t-z / c$ at the point $z$, as the time interval from the time the end of the non-scattered part of the exciting pulse has reached this point. This is the time measured experimentally in SR experiments. Under this substitution, the derivatives become

$$
\frac{\partial}{\partial t} \rightarrow \frac{\partial}{\partial t^{\prime}}, \quad \frac{\partial}{\partial z}+\frac{1}{c} \frac{\partial}{\partial t} \rightarrow \frac{\partial}{\partial z}
$$

and eqs. (7) and (8) for the amplitudes of the charge displacement and the electromagnetic field become

$$
\begin{aligned}
\frac{\partial \mathcal{X}\left(t^{\prime}, z\right)}{\partial t^{\prime}}+\frac{\gamma}{2} \mathcal{X}\left(t^{\prime}, z\right) & =\frac{-i q}{2 m \omega_{0}} \mathcal{E}\left(t^{\prime}, z\right) \\
\frac{\partial \mathcal{E}\left(t^{\prime}, z\right)}{\partial z} & =\frac{-i \omega_{0} c \mu_{0} \rho_{e}}{2} \mathcal{X}\left(t^{\prime}, z\right)
\end{aligned}
$$

The uncoupled amplitude equations. - We introduce the lifetime of the excited level of the nuclei $\tau_{0}=1 / \gamma$, dimensionless resonant frequency $w_{0}=\omega_{0} \tau_{0}$ and dimensionless time delay $\tau=t^{\prime} / \tau_{0}=\gamma t^{\prime}$. To eliminate the term with $\gamma$, in eq. (9) we introduce the substitutions

$$
\mathcal{X}(\tau, z)=e^{-\frac{\tau}{2}} \tilde{\mathcal{X}}(\tau, z), \quad \mathcal{E}(\tau, z)=e^{-\frac{\tau}{2}} \tilde{\mathcal{E}}(\tau, z) .
$$


With these substitutions, eqs. (9) and (10) reduce to the system

$$
\begin{aligned}
\frac{\partial \tilde{\mathcal{X}}(\tau, z)}{\partial \tau} & =\frac{-i q}{2 m \omega_{0} \gamma} \tilde{\mathcal{E}}(\tau, z), \\
\frac{\partial \tilde{\mathcal{E}}(\tau, z)}{\partial z} & =\frac{-i \omega_{0} c \mu_{0} \rho_{e}}{2} \tilde{\mathcal{X}}(\tau, z) .
\end{aligned}
$$

It is obvious from these equations that $\mathcal{E}_{R e}(\tau, z)$, the real part of the amplitude of the electromagnetic field $\mathcal{E}(\tau, z)$, is related to $\mathcal{X}_{\operatorname{Im}}(\tau, z)$, the imaginary part of the amplitude of the charge displacement $\mathcal{X}(\tau, z)$, and vice versa. This means that at each point in time and space there is a $\pi / 2$ phase shift between the electromagnetic field and the charge vibrations.

The nuclei, excited by the exciting pulse entering the medium vibrate and produce oscillating currents which generated a primary delayed coherent field. We associate the amplitude of this primary field with $\tilde{\mathcal{E}}_{R e}(\tau, z)$. This primary field, in turn, also excites the nuclei and generates a secondary field. Since the radiation field of a non-relativistic moving charge is proportional to its acceleration [12], it follows that the phase of this radiation field is opposite to the phase of the vibrating nuclei. Hence, there exists a $-\pi / 2$ phase shift between the primary field and the secondary radiation fields. This requires one to consider an imaginary amplitude. We associate the amplitude of the secondary field with $\tilde{\mathcal{E}}_{I m}(\tau, z)$.

Combining eqs. (12) and (13) and denoting the linear absorption coefficient at resonance by

$$
\mu_{r}=\frac{c \mu_{0} q \rho_{e}}{4 m \gamma}
$$

we can decouple the above system and obtain the two (identical) second-order equations

$$
\frac{\partial^{2} \tilde{\mathcal{X}}(\tau, z)}{\partial z \partial \tau}=-\mu_{r} \tilde{\mathcal{X}}(\tau, z)
$$

and

$$
\frac{\partial^{2} \tilde{\mathcal{E}}(\tau, z)}{\partial \tau \partial z}=-\mu_{r} \tilde{\mathcal{E}}(\tau, z)
$$

for $\tilde{\mathcal{X}}(\tau, z)$ and $\tilde{\mathcal{E}}(\tau, z)$.

The solution for the electromagnetic field. - In order to obtain the formula for the delayed electromagnetic field $E(t, z)$ we calculate $\tilde{\mathcal{E}}_{R e}(\tau, z)$ and $\tilde{\mathcal{E}}_{I m}(\tau, z)$ separately.

Firstly, we obtain $\tilde{\mathcal{E}}_{R e}(\tau, z)$. To do this, we solve first eq. (15) for the amplitude $\tilde{\mathcal{X}}_{I m}(\tau, z)$ and then use eq. (12). To obtain a unique solution we need to consider boundary and initial conditions.

At the entrance of the resonant medium, $z=0$, it is natural to assume that the amplitude $\mathcal{X}_{I m}(\tau, 0)$ is decaying with damping factor $\gamma$, since there is no external field at this position for $\tau>0$. This implies that $\tilde{\mathcal{X}}_{I m}(\tau, 0)=X_{0}$ for some positive constant $X_{0}$.
We further assume that the medium is thin enough so that the exciting pulse is essentially unattenuated and thus appears identical to each nucleus. Since at time $\tau=0$ the exciting pulse has already passed the point $z$ and has excited the nuclei in the medium, the amplitude of the vibrations is the same at any position (including $z=0$ ), hence requiring $\mathcal{X}_{I m}(0, z)=\tilde{\mathcal{X}}_{I m}(0, z)=X_{0}$.

Equation (15) together with the conditions $\tilde{\mathcal{X}}_{I m}(\tau, 0)=$ $X_{0}$ and $\tilde{\mathcal{X}}_{I m}(0, z)=X_{0}$ can be turned into the integral equation

$$
\tilde{\mathcal{X}}_{I m}(\tau, z)=X_{0}-\mu_{r} \int_{0}^{\tau} \mathrm{d} \tau^{\prime} \int_{0}^{z} \tilde{\mathcal{X}}_{I m}\left(\tau^{\prime}, z^{\prime}\right) \mathrm{d} z^{\prime} .
$$

This equation can be solved iteratively by defining $\tilde{\mathcal{X}}_{I m}^{(0)}(\tau, z)=X_{0}$ and using the scheme

$$
\tilde{\mathcal{X}}_{I m}^{(n+1)}(\tau, z)=X_{0}-\mu_{r} \int_{0}^{\tau} \mathrm{d} \tau^{\prime} \int_{0}^{z} \tilde{\mathcal{X}}_{I m}^{(n)}\left(\tau^{\prime}, z^{\prime}\right) \mathrm{d} z^{\prime} .
$$

By induction, one can easily show that

$$
\tilde{\mathcal{X}}_{I m}^{(n)}(\tau, z)=X_{0} \sum_{j=0}^{n} \frac{\left(-\mu_{r} \tau z\right)^{j}}{(j !)^{2}}
$$

which converges to

$$
\tilde{\mathcal{X}}_{I m}(\tau, z)=X_{0} \sum_{j=0}^{\infty} \frac{\left(-\mu_{r} \tau z\right)^{j}}{(j !)^{2}}
$$

The infinite sum on the right-hand side is the power series expansion of $J_{0}\left(\sqrt{4 \mu_{r} \tau z}\right)$, the Bessel function of the first kind of order zero with argument $\sqrt{4 \mu_{r} \tau z}$. Hence,

$$
\tilde{\mathcal{X}}_{I m}(\tau, z)=X_{0} J_{0}\left(\sqrt{4 \mu_{r} \tau z}\right),
$$

which upon substitution into eq. (12) and use of the identity $J_{0}^{\prime}(x)=-J_{1}(x)$, yields

$$
\tilde{\mathcal{E}}_{R e}(\tau, z)=A\left(4 \mu_{r} z\right) J_{1}\left(\sqrt{4 \mu_{r} \tau z}\right) / \sqrt{4 \mu_{r} \tau z}
$$

with the constant $A=X_{0} m \omega_{0} \gamma / q$. Thus, the amplitude of the primary delayed field is given by

$$
\mathcal{E}_{R e}(\tau, z)=A e^{-\frac{\tau}{2}}\left(4 \mu_{r} z\right) J_{1}\left(\sqrt{4 \mu_{r} \tau z}\right) / \sqrt{4 \mu_{r} \tau z} .
$$

It is worthwhile to note in passing that the intensity (the square of the amplitude) of this component of the electromagnetic field is proportional to

$$
e^{-\tau}\left(4 \mu_{r} z\right)^{2} \frac{J_{1}^{2}\left(\sqrt{4 \mu_{r} \tau z}\right)}{4 \mu_{r} \tau z}
$$

which, at the exit point $z=d$, yields the time spectrum (2) for the pulse propagation in a single resonant medium with effective thickness $T=4 \mu_{r} d$, cited in the literature.

We wish to emphasise however, that formula (19) has two shortcomings. Firstly, it gives rise to a time spectrum which has values close to zero corresponding to the zeros 
of $J_{1}$, which are not observed experimentally. Secondly, it predicts a zero field at the entrance $z=0$ of the medium which excludes the existence of backscattering. Coherent backscattering occurs when coherent radiation propagates through a medium which has a large number of scattering centers. It was observed experimentally and justified theoretically, see, for example, [7] and [8]. A secondary delayed field which is non-zero at the entrance and with zeros at different locations resolves these shortcomings. Indeed, as we will show later, the time spectrum obtained by the use of this secondary field fits well the experimental data. This is an indication that coherent backscattering is also present in our situation and suggests further experimental investigation to fully understand its physical meaning and origin.

We now obtain $\mathcal{E}_{\operatorname{Im}}(\tau, z)$ associated with the secondary field. It is natural to assume that this field has the same damping as that of the charge vibrations. So, the boundary condition is $\mathcal{E}_{\operatorname{Im}}(\tau, 0)=E_{0} e^{-\frac{\tau}{2}}$ for some constant $E_{0}=\tilde{\mathcal{E}}_{I m}(\tau, 0)$.

Furthermore, for any given point $z_{0}$ in the medium, the radiation field at time $t$ results from the contributions of all the nuclei whose world line cross the backward light cone with vertex at $\left(t, z_{0}\right)$. Since at time $\tau=0$, the front and most of the pulse have already excited all the nuclei in the interval $0<z<d$ and thus contribute to this field and coherence is preserved in both forward and backward directions, we postulate that $\mathcal{E}_{I m}(0, z)$ is the same at any position and thus $\mathcal{E}_{I m}(0, z)=\tilde{\mathcal{E}}_{I m}(0, z)=E_{0}$. Furthermore, $E_{0}$ is proportional to $d$. This is also consistent with the fact that if $d$ approaches zero there is no secondary radiation field. Since the radiation field is proportional to the average acceleration $X_{0} \omega_{0}^{2} / 2$ and the charge density of these excited nuclei $\rho_{e}$, we write $E_{0}=b X_{0} \omega_{0}^{2} \rho_{e} d /\left(2 \varepsilon_{0} c^{2}\right)$ for some constant $b$ of units of length ${ }^{-1}$.

Using the same method as the one for the calculation of $\tilde{\mathcal{X}}_{I m}(\tau, z)$ we obtain

$$
\tilde{\mathcal{E}}_{I m}(\tau, z)=E_{0} J_{0}\left(\sqrt{4 \mu_{r} \tau z}\right)
$$

Combining eqs. (6), (11), (18) and (21), we obtain the formula for the total delayed field of a coherent SR pulse propagating through a single resonant medium

$$
\begin{aligned}
E(\tau, z) & =e^{\left(i w_{0}-1 / 2\right) \tau} \\
& \times\left(A\left(4 \mu_{r} z\right) \frac{J_{1}\left(\sqrt{4 \mu_{r} \tau z}\right)}{\sqrt{4 \mu_{r} \tau z}}+i E_{0} J_{0}\left(\sqrt{4 \mu_{r} \tau z}\right)\right) .
\end{aligned}
$$

Note that this formula predicts both backscattering and a non-zero field at the zeros of the primary field.

The time spectrum. - Introducing the effective thickness $T=4 \mu_{r} d$ and $\alpha=E_{0} /(A T)=b \omega_{0} / c$, the exiting field (the time response of the delayed resonant pulse)

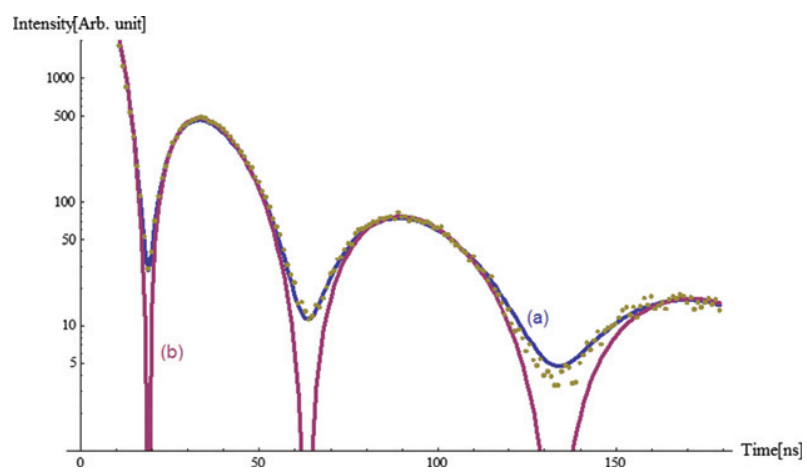

Fig. 1: (Colour on-line) Comparison of the experimental time spectrum of a $12 \mu \mathrm{m}$ enriched stainless-steel foil with (a) the theoretical curve given by (24), (b) one-term formula (2) and the experimental data (dots).

is proportional to

$$
E(\tau, T) \propto e^{\left(i w_{0}-1 / 2\right) \tau} T\left(\frac{J_{1}(\sqrt{T \tau})}{\sqrt{T \tau}}+i \alpha J_{0}(\sqrt{T \tau})\right)
$$

The time spectrum of a pulse propagating through a resonant medium is the field intensity of the pulse at the exit $I(\tau)=|E(\tau, d)|^{2}$. Using (23), we obtain

$$
I(\tau) \propto e^{-\tau} T^{2}\left(\frac{J_{1}^{2}(\sqrt{T \tau})}{T \tau}+\alpha^{2} J_{0}^{2}(\sqrt{T \tau})\right),
$$

where the constant $\alpha=b \omega_{0} / c$ depends only on the properties of the medium. We compared our formula using the effective thickness $T=109$, lifetime of the excited level $\tau_{0}=141.11 \mathrm{~ns}$ and $\alpha=0.038$, with the experimental time spectrum of an enriched ${ }^{57} \mathrm{Fe}$ stainless-steel foil obtained in an experiment under the leadership of Ralf Röhlsberger (2014) at PETRAIII, see fig. 1. Our formula fits the experimental data much better than the usual one-term formula in $(2)$.

Nowadays, the known computer programs MOTIF [9] and CONUSS [10] are commonly used to evaluate and fit the experimental time spectra. For a single-line target and for a given thickness parameter $T$ these programs reproduce the one-term formula time spectrum (formula (24) with $\alpha=0$ ), but in order to achieve the same quality fit [13] like the one predicted by our formula it is necessary to average the thickness parameter over a significant range.

This can be also explained mathematically as follows. Changing the thickness parameter $T$ by a small amount $\Delta T$ will effectively change the exiting field $E(\tau, T)$ by an amount $\Delta E=(\partial E(\tau, T) / \partial T) \Delta T$. From (23) for $E_{0}=$ $0=\alpha$ the exiting field is

$$
E(\tau, T)=A e^{\left(i w_{0}-1 / 2\right) \tau} T \frac{J_{1}(\sqrt{T \tau})}{\sqrt{T \tau}} .
$$


Thus,

$$
\frac{\Delta E}{\Delta T}=A e^{\left(i w_{0}-1 / 2\right) \tau}\left(\frac{J_{1}(\sqrt{T \tau})}{\sqrt{T \tau}}+T \frac{\partial}{\partial T}\left(\frac{J_{1}(\sqrt{T \tau})}{\sqrt{T \tau}}\right)\right) .
$$

Introducing $\zeta=\sqrt{T \tau}$ and using the identities $\left(\zeta^{-1} J_{1}(\zeta)\right)^{\prime}=-\zeta^{-1} J_{2}(\zeta), J_{2}(\zeta)=2 J_{1}(\zeta) / \zeta-J_{0}(\zeta)$ and $\frac{\partial \zeta}{\partial T}=\tau / 2 \zeta$ we obtain

$$
T \frac{\partial}{\partial T}\left(\frac{J_{1}(\sqrt{T \tau})}{\sqrt{T \tau}}\right)=-\frac{J_{1}(\sqrt{T \tau})}{\sqrt{T \tau}}+\frac{1}{2} J_{0}(\sqrt{T \tau}) .
$$

Thus,

$$
\Delta E=A e^{\left(i w_{0}-1 / 2\right) \tau} \frac{1}{2} J_{0}(\sqrt{T \tau}) \Delta T .
$$

If we assume that the distribution of $\Delta T$ is symmetric with respect to the true value of $T$, the averaging of the thickness parameter produces the additional term in (24).

Conclusions. - A new formula (22) for the delayed electromagnetic field and the time spectrum (24) of a coherent synchrotron radiation pulse propagating through a single resonant medium is obtained. Following [4], the delayed field was obtained by solving simultaneously the coupled equations (4) and (5) describing the interaction of the electromagnetic field and the oscillating currents in the medium. Using the slow-varying amplitude modification, introducing the time delay and extracting the damping we reduced the problem to a first-order coupled system and then to two second-order uncoupled equations for the complex amplitudes of the field and charge displacement.

We have further separated the primary field (with the real part of the amplitude of delayed field) generated by the oscillating currents caused by the exciting pulse from the secondary field (with the imaginary part of the amplitude) resulting from the charge vibrations caused by the primary radiation field. The secondary field was calculated under the assumptions that the initial SR pulse instantly creates a collective nuclear excitation extending over all the parts of the medium and that coherence is preserved in both forward and backward directions. Unlike the commonly accepted one-term formula for such a time spectrum, our formula contains an additional term due to the secondary radiation field. Our final formula (22) for the total delayed field allows for both backscattering (observed experimentally and explained theoretically) and a non-zero field at the zeros of the primary field (confirmed by the experimental time spectra).
The commonly known computer programs MOTIF and CONUSS, used nowadays to evaluate and fit experimental time spectra, produce for a single resonant medium, the same spectrum as the one predicted by the one-term formula (2). However, in order to achieve a good fit to the experimental spectrum they require the averaging of the thickness parameter over a significant range. Using the experimental data from the time spectrum of an enriched stainless-steel foil obtained recently at PETRA III as a test case, our new formula provides the same quality fit without the need for such averaging. This comes as no surprise. We show mathematically that the averaging of the thickness parameter in the one-term formula produces in fact the additional term in our new formula.

$$
* * *
$$

Supported in part by the German-Israeli Foundation for Scientific Research and Development: GIF No. 1078107.14/2009. We wish to thank Hans-Christian Wille for providing the fit of the data with CONUSS and Gennady V. Smirnov, Ralf Röhlsberger and Yuri V. SHVYD'KO for their stimulating remarks.

\section{REFERENCES}

[1] Lynch F. J., Holland R. E. and Hamermesh M., Phys. Rev., 120 (1960) 513.

[2] Smirnov G. V., Hyperfine Interact., 123/124 (1999) 31.

[3] Kagan Yu., Afanas'ev A. M. and Kohn V. G., J. Phys. C: Solid State Phys., 12 (1979) 615.

[4] Burnham D. C. and Chiao R. Y., Phys. Rev., 188 (1969) 667.

[5] Smirnov G. V., Hyperfine Interact., 97/98 (1996) 551; Shvyd'ko Y. V., Phys. Rev. B, 59 (1999) 9132; Конм V. G. and Smirnov G. V., Phys. Rev. B, 76 (2007) 104438; Smirnov G. V. et al., Phys. Rev. A, 76 (2007) 043811.

[6] VAN BurCK U., Hyperfine Interact., 123/124 (1999) 483.

[7] Akkermans E., Wolf P. E. and Maynard R., Phys. Rev. Lett., 56 (1986) 1471.

[8] Wiersma D. S., van Albada M. P. and LagendiJk A., Phys. Rev. Lett., 75 (1995) 1739.

[9] SHVID'ko Yu., Hyperfine Interact., 125 (2000) 173.

[10] Sturhahn W., Hyperfine Interact., 125 (2000) 149.

[11] Boyd R. W., Nonlinear Optics (Academic Press) 2008, p. 493.

[12] JaCkson J. D., Classical Electrodynamics (Wiley) 1998, p. 675 .

[13] Willle H. C., private communication (2014). 